\title{
The TMS Foundation: Success in 2018
}

\section{Kaitlin Calva}

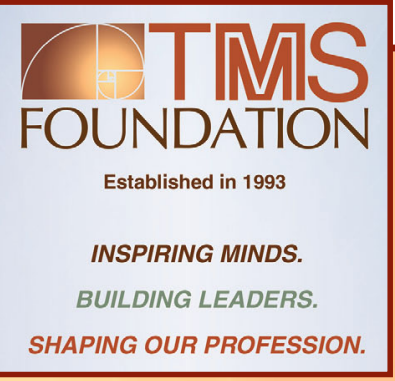

The story of the TMS Foundation's 25th anniversary year is a good one. Considerable strides have been made enhancing the profile of the Foundation and spreading the word about its good work among the wider TMS membership - progress that is reflected in our increasing number of donors and donations. In 2018, the Foundation established a new endowed award, continued supporting a variety of programs, and, taking into account all of its fundraising and revenue sources, had one of its best years financially since its revitalization began in 2013. The 2018 year-end appeal reached its goal and raised more than $\$ 100,000$ by the campaign's conclusion on December 31, 2018. Altogether, 374 generous individuals made contributions, including 84 new donors for the yearthat's up from 2017's 325 and 74 donors, respectively. Overall, the TMS Foundation raised \$513,721 last year and saw its highest number of annual donors in the last five years.

In addition to the highly visible year-end appeal, the Foundation took on new ventures and expanded others throughout the year, such as the William D. Nix Award. Endowed and fully funded by Nix's Ph.D. students in recognition of the tremendously positive impact he has had on their lives, the new Society-level honor will confer its first award in 2020.

In addition, the first recipients of the Acta Materialia Inc. Undergraduate Scholarship were announced in

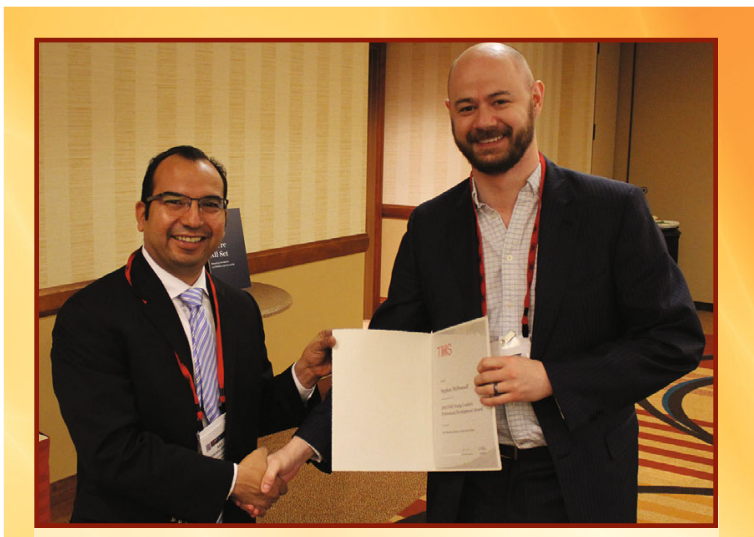

Raymundo Arroyave (left), Functional Materials Division (FMD) chair, presents Stephen McDonnell (right), assistant professor at the University of Virginia, with the 2018 FMD Young Leaders Professional Development Award during TMS2018.

2018. TMS members were eager to answer the call and support their technical homes within TMS during a campaign to grow the division endowments that house TMS's Technical Division Scholarships. And the Society continued its support of the future of the minerals, metals, and materials community through a gift to the TMS Foundation.

On other fronts, the Foundation maintained its commitment to advancing STEM (science, technology, engineering, and mathematics) initiatives by lending a financial hand to both the Materials Explorers ${ }^{\mathrm{TM}}$ program and the TMS Bladesmithing Competition. It also continued to fund a number of other scholarships for deserving students, leadership development programs for early career individuals, and prestigious honors recognizing talented professionals, including TMS's two diversity and inclusion awards.

"So, five years into its revitalization, and the Foundation is sustaining its growth quite successfully," said Garry W. Warren, TMS Foundation Board of Trustees chair. "Thank you to all who not only made our 25th anniversary a year to celebrate, but who also shared the Foundation's story and helped us build our image. Our success is not possible without your generosity and ongoing commitment to securing the future of our profession."

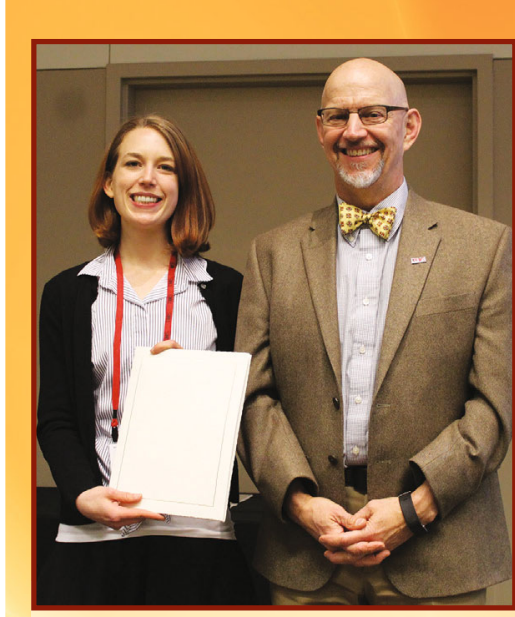

Cayla Harvey (left), University of Nevada - Reno, receives the 2018 Structural Materials Division (SMD) Scholarship from SMD vice-chair Daniel Miracle (right) at the TMS 2018 Annual Meeting \& Exhibition (TMS2018).

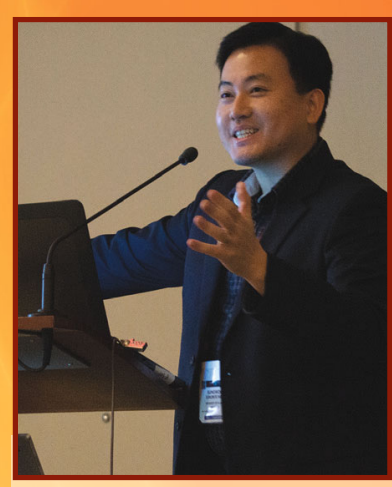

Sung Woo Nam, assistant professor at the University of Illinois, presented a lecture as one of the two 2018 TMS Early Career Faculty Fellows during the Young

Professional Tutorial Luncheon held at TMS2018. 


\section{Legacy Circle}

This society recognizes individuals who have provided for the Foundation through planned giving.

Lucinda and Martin Glicksman

Garry W. Warren and Larry Goldman

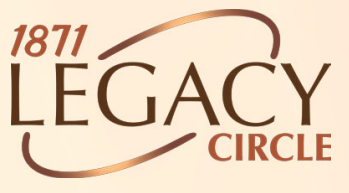

\section{Lifetime Giving Honorific Societies}

\section{Diamond Society} (\$100,000 or more)

Lucinda and Martin Glicksman

Geraldine McCulley Wadsworth and Jeffrey Wadsworth

\section{Platinum Society} (\$50,000 to \$99,999)

Robyn and Rob Wagoner

\section{Gold Society} (\$20,000 to \$49,999)

Seta and Diran Apelian

Om P. Arora (deceased)

Carl M. Cady

Linda and Lionel Kimerling

Kenneth R. Kinsman

Marc A. Meyers

Garry W. Warren and Larry Goldman

\section{Titanium Society} (\$10,000 to \$19,999)

Joseph D. Defilippi

Gordon H. Geiger

Lorenzo Martinez Gomez

Maria Oliva and Kevin Hemker

Michelle A. and Edward D. Herderick

Elizabeth Holm and David Crockett

Carol and Stanley Howard

Diane and David Matlock

Tina and Daniel Miracle

Deepa and Brajendra Mishra

Tai-Gang Nieh

Warren Oliver

Sonia and Deepankar Pal

Marilyn and George Pharr

David Alan Shifler in Memory of Robert B. Pond Sr.

Michèle and Patrice Turchi

William G. Wagstaff (deceased)

Deborah L. Yaney
Silver Society (\$5,000 to $\$ 9,999)$

Viola L. Acoff

Cynthia K. Belt

Cynthia Bognar

Joan and David DeYoung Jean and William Nix

James Earthman

Carol and Kenneth Fuchs

Jeffery C. Gibeling

Stacy and Bob Gleixner

George T. Gray III

William C. Harrigan Jr.

Hani Henein

Joy and Gregory Hildeman

George Krauss

Diane and David Laughlin

Thaddeus B. Massalski

Ronald E. Miller

Michael J. Mills

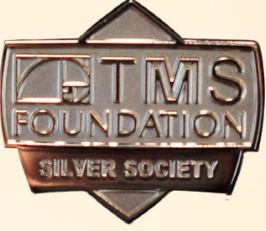
Harold W. Paxton

Ray D. Peterson

Lynne and James Robinson Wolfgang A. Schneider Angela and Alexander Scott Mary C. and Robert D. Shull King-Ning Tu

Frank E. Wagstaff

Robert B. Wagstaff

Leigh and Timothy Weihs

Wendelin J. Wright and John C. Bravman

\section{The 2018 TMS Foundation Honor Roll}

The TMS Foundation thanks the following TMS members and friends for their generous support in 2018. Their gifts are critical to the success of current and future TMS Foundation programs, and therefore critical to advancing the future of the minerals, metals, and materials science and engineering community.

To secure your place on the 2019 TMS Foundation Honor Roll, visit www.TMSFoundation.org/Contribute to make an online donation. Or contact TMS Foundation staff at TMSFoundation@tms.org or 1-724-776-9000 with questions or for more information on additional donation options. 


\section{Annual Giving Honor Roll}

\section{Foundation Leader (\$2,500 or more)}

Aurubis AG

Bognar and Company Inc.

Carl M. Cady in Memory of Cathy

LeClaire

Joseph D. Defilippi

Dyrkacz Family Charitable Trust

in Memory of Mary and W.

William Dyrkacz

James Earthman

Carol and Kenneth Fuchs

Rogelio Gasca Neri

Marsha and Jeffery Gibeling

Veronique T. Gillard

Stacy and Bob Gleixner

Lucinda and Martin Glicksman

Lorenzo Martinez Gomez

Steven H. Goods

William C. Harrigan Jr.

Maria Oliva and Kevin Hemker

Michelle A. and Edward

D. Herderick

The Charles \& Esther Lee

Kimerling Charitable

Foundation

Kenneth R. Kinsman

Diane and David Matlock

Marc A. Meyers

Michael J. Mills

Tina and Daniel Miracle

Deepa and Brajendra Mishra

Tai-Gang Nieh

Jean and William Nix

Warren Oliver

Sonia and Deepankar Pal 40/40cus

Marilyn and George Pharr

David Alan Shifler in Memory of

Robert B. Pond Sr.

Robyn and Rob Wagoner

Jian-Sheng Wang

Leigh and Timothy Weihs
Wendelin J. Wright and John

C. Bravman

Deborah L. Yaney

Foundation Founder

(\$1,000 to \$2,499)

American Institute of Mining,

Metallurgical and Petroleum

Engineers (AIME)

Nancy and Iver Anderson

Anonymous

Corbett C. Battaile

David L. Bourell

Bryan A. Chin

Amy and Kester Clarke

Joan and David DeYoung

James C. Foley

Ellen S. and Paul S. Gilman

Gopher Resource LLC

George T. Gray III

Hani Henein

Andrea and Nate Holland

Yong-Suk Kim

Kristen and Paul Kitabjian

Diane and David Laughlin

Seok-Woo Lee

Michele V. Manuel 40/40cus

Thaddeus B. Massalski

Mary and Bob Minardi

Leah and Paul Ohodnicki 40/40cus

Harold W. Paxton

Ray D. Peterson

Lynne and James Robinson

Alexander and Angela Scott

Mary C. and Robert D. Shull

Raymond L. Smith (deceased)

Ann and Dan Thoma

King-Ning Tu

Michèle and Patrice Turchi

Wagstaff Inc.

Garry W. Warren and Larry Goldman

Steven J. Zinkle

\section{Foundation Builder}

(\$500 to \$999)

Acta Materialia Inc.

Anonymous

Arief Budiman

Carelyn Campbell and Mark

Stoudt

Maxwell Choi

Cherqueta R. Claiborn 40/40c cus

Mary and Ray Decker

Adrian C. Deneys in Honor of Jarek Drelich

Tom Dragone

Jeffrey Florando

Seung Min Han 40/40aub

Carolyn M. Hansson

Siegfried S. Hecker

John A. Howarter 40/40clus

George Krauss

Erica Thea Lilleodden

Ramulu Mamidala

Paul Mason

III Ryu

Wolfgang A. Schneider

James L. Smith

Brian G. Thomas

Gregory B. Thompson

Michael D. Uchic

Natalie and Chester J. Van Tyne

James A. Yurko

Thermo-Calc Software Inc. (U.S.)

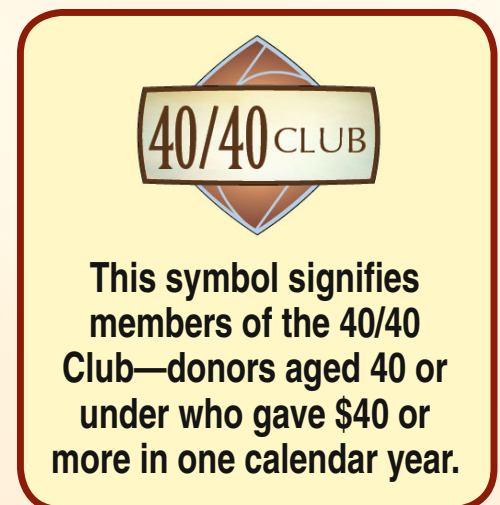




\section{Foundation Provider (\$250 to \$499)}

John E. Allison

Raymundo Arroyave

Sudarsanam S. Babu

Thomas P. Battle

Cynthia K. Belt

Megan Brewster 40/40cuB

Gang Feng

Joy H. Forsmark

Ronald Gibala

William P. Imrie

Sung K. Kang

Sharvan Kumar

Alexis C. Lewis

Krishnan K. Sankaran

Kathy and George Spanos

Thermo-Calc Software AB

(Sweden)

Joseph F. Thomas Jr.

\section{Foundation}

Ambassador

(\$100 to \$249)

Reza Abbaschian

Ian Baker

Robert J. Beltz

Thomas R. Bieler

John C. Bierlein

William J. Boettinger

Brad L. Boyce

John M. Brear

Kimberly and Patrick Cannon

Tadeu Carneiro

Joseph Chieu

Julie A. Christodoulou

Harold R. Clark

Megan J. Cordill 40/40cub

Subodh K. Das

Alfredo Oscar Del Campo

Ronald J. Dimelfi

Jaroslaw W. Drelich

Alan P. Druschitz

Jeffrey W. Fergus
Stephen M. Foiles

Alois J. Franke

Brent T. Fultz

Amber Lynn Genau
Anthony F. Giamei

Julia R. Greer

Ola L. Harrysson

Jeffrey A. Hawk

Richard W. Hertzberg

John P. Hirth

Lee E. Hoffman

Douglas C. Hofmann

Michael E. Kassner

Peter L. Kern

Matthew J. Kramer

Jessica A. Krogstad 40/40cub

Jamie J. Kruzic

Alan Lee

Sanboh Lee

Carlos G. Levi

Yong Chae Lim

Scott J. Litzelman

Alan A. Luo

Koichiro Mashio

Alexander McLean

Terry R. McNelley

Edward E. Mild

Thomas W. Montemarano

Neville R. Moody

John E. Morral

Jagdish Narayan

G. Robert Odette

Suzanne and André B. Phillion

Norbert L. Piret

Paul D. Prichard

Ramana G. Reddy

Anthony D. Rollett

Herman S. Rosenbaum

Kirk Alan Rogers

Adrian S. Sabau

Mary Samsa

Georg J. Schmitz

Erin and Justin Scott 40/40сcü

James W. Sears
Doug Shymoniak 40/40cus

T.S. Srivatsan

Malcolm Stocks

Dilip K. Subramanyam

Chantal K. Sudbrack

Ziqi Sun $40 / 40$ c

Arthur P. Turner

Pello Uranga

Dirk E. Verhulst

Richard M. Waterstrat

William J. Weber

John Randolph Wood

Janelle P. Wharry

David B. Witkin

Clarissa Yablinsky and

Benjamin Morrow

40/40caub

Baojun Zhao

Yuntian T. Zhu

\section{Foundation Patron}

(\$25 to \$99)

Johannes Aalbu

Vincent Ben B. Abratigue

Patrick M. Afenya

Abdul Ganiyu F. Alabi

Anonymous

Anonymous

Gerardo Raul Alvear Flores

Ronald W. Armstrong

Raiyomand F. Aspandiar

David F. Bahr

Andrew H. Baker

Eduardo Balladares

Anthony John Barnes

David L. Baty

Irene J. Beyerlein

Christine J. Beyke

George J. Binczewski

Michael P. Brady

Kyle S. Brinkman

Douglas Burkes

Ronald N. Caron

$40 / 40 \mathrm{CLUB}$

Teresa Carvajal

Ellen K. Cerreta 


\begin{tabular}{|c|c|c|}
\hline Indrajit Charit & Hojong Kim & Linda L. Rishel \\
\hline Deep Choudhuri & Randolph E. Kirchain & Michael Roesner-Kuhn \\
\hline Leo Christodoulou & Sanji Kitaoka & Joseph C. Sabatini \\
\hline Blythe Gore Clark 40/40aus & Paul Korinko & Ramachandran \\
\hline Danielle L. Cote & Dileep Kumar C J & Sankarasubramanian \\
\hline Jacob Crane & Bennett C. Larson & W. Joseph Schlitt \\
\hline Herbert Danninger & Rodolfo S. Lazo Davila & Jan-Marten Seitz \\
\hline Steven L. Dedmon & Jae-chun Lee & Jeffrey E. Shield \\
\hline Shen J. Dillon 40/40cus & John J. Lewandowski & Amit Shyam \\
\hline Roger D. Doherty & Jian Li & Eugene A. Silva \\
\hline Curtiss Otto Ehrsam & Pei Yong Li & Alok Singh \\
\hline Abdallah K. Elsayed & Yan Li & Ernest J. Sirois \\
\hline Michael G. Fahrmann & Yi Liu & James L. Smialek \\
\hline John W. Fernihough & Stefan Luidold & John G. Speer \\
\hline Robert D. Field & Juan Carlos Madeni & Keith W. Sweatman \\
\hline Joel Filner & Satish C. Manaktala & Eric M. Taleff \\
\hline Paul S. Follansbee & Peter E. Marshall & John M. Tartaglia \\
\hline Tim Gabb & Jan W. Matousek & Karem Tello \\
\hline Gordon H. Geiger & Jyotirmoy Mazumder & Peter-Hans ler veer \\
\hline William W. Gerberich & David L. McDowell & Canos IN. IOme \\
\hline Ashok Kumar Gogia & Masahiro Michino & Merk A Tcrhonn \\
\hline Robert J. Gottschall & Arthur E. Morris & Wrark A. I Scriopp \\
\hline Richard E. Grace & Carol E. Moyer & Paul R. Van Houtte \\
\hline John A.S. Green & Erik M. Mueller 40/40cus & Christelle E. Van Rensburg \\
\hline Xiaofei Guan & Dmitry Nekrashevich & Lucio B. Vazquez \\
\hline Mustafa Guclu & Sergey B. Novichkov & Heather M. Volz \\
\hline Douglas C. Haag & Funsho K. Ojebuoboh & Paulo Von Kruger \\
\hline Frederick R. Hafner & Elsa Olivetti 40/40c c1uB & Guntram B. Wagner \\
\hline Adam J. Hehr 40/40 a cus & Dmytro Orlov & Cong Wang $\quad 40 / 40^{\circ}$ \\
\hline Richard G. Hennig & Harmen Oterdoom & Thomas Peter Wertli \\
\hline Andreas Holzenburg & Yuan Pang & Charles V. White \\
\hline Peter Hosemann 40/40счиB & Anita Patricio & Glenn G. Whiteside \\
\hline Robert W. Hyers & Edward A. Pchola & James R. Wilcox \\
\hline Masahiko lkeda & Jianping Peng 40/40club & Matthew A. Willard \\
\hline Laurence A. Jackman & Evelyn M. Putman & Edward McRae Williams \\
\hline Melvin R. Jackson & V. Ramachandran & Winnie K. Wong-Ng \\
\hline Gregg M. Janowski & Ramesh C. Rao & Patrick Woolsey \\
\hline Frank Johnson & Arne P. Ratvik & Sisi Xiang \\
\hline Andrea Jokisaari & Petra Reinke & Jian-hong Yang \\
\hline J. Brian Jordon 40/40c ü & Yu Ren & Jien-Wei Yeh \\
\hline Karl Ulrich Kainer & Charles E.C. Rense & Hung-Wei Yen \\
\hline Tomoyuki Kakeshita & Steve Reubi & Charles Fred Yolton \\
\hline Marian S. Kennedy 40/40c CuB & Lewis Reynolds & Qiaoshi Zeng \\
\hline Mark W. Kennedy & Orlando Rios 40/40caus & Jiang Zheng \\
\hline
\end{tabular}

Linda L. Rishe

Michael Roesner-Kuhn

Joseph C. Sabatin

Ramachandran

W. Joseph Schlitt

Jan-Marten Seitz

Jeffrey E. Shield

mit Shyam

Alok Singh

Ernest J. Sirois

Keith W. Sweatman

Karem Tello

Carlos N. Tome

Stewart W. Towle

A. Tschopp

Ugbo

Paul R. Van Houtte

作

B. Vazquez

Heather M. Volz

untram B. Wagne

Cong Wang 40/40ciun

Thomas Peter Wertl

Charles V. White

Glenn G. Whiteside

Matthew A. Willard

Edward McRae Williams

$\mathrm{g}-\mathrm{Ng}$

Patrick Woolsey

ny Yang

Hung-Wei Yen

Charles Fred Yolton

Qiaoshi Zeng

Zheng 